\section{Pulmonary artery sarcoma mimicking chronic pulmonary} thromboembolism

Sarcoma da artéria pulmonar simulando tromboembolismo pulmonar crônico

\section{Dear Editor,}

A 35-year-old woman was admitted in our institution with a 2-year history of dyspnea, hemoptysis and chest pain. Chest computed tomography (CT) demonstrated filling defects in the right pulmonary artery and some of its branches (Figure 1A). Transthoracic echocardiography showed right heart chambers enlargement and increased pulmonary artery systolic pressure. These test results associated to the patient's clinical history, allowed for the diagnosis of chronic pulmonary thromboembolism (PTE).

After six months of treatment without clinical improvement, a new contrast enhanced CT revealed a growing intraluminal filling defect and a lobulated mass on the right pulmonary artery and its branches, with areas of contrast enhancement (Figure 1 - B,C,D). In addition to the CT findings, magnetic resonance imaging identified restriction of water diffusion. These imaging findings yielded the diagnosis of pulmonary artery sarcoma (PAS).

A significant clinical worsening was observed and the patient died before she could be submitted to a diagnostic/therapeutic surgical procedure.
Vascular lesions of the chest have not been frequently described in the Brazilian radiological literature ${ }^{(1-5)}$. PAS is a rare malignant tumor that develops from mesenchymal cells in the intima of the pulmonary artery ${ }^{(6)}$. In general, it affects the central pulmonary arteries, close to the pulmonary valve ${ }^{(7)}$, resulting in significant morbidity and high mortality rates ${ }^{(8)}$. There is no predilection for sex, occurring most commonly in the fifth decade of life $^{(9)}$

In general, symptoms are nonspecific with dyspnea, cough, hemoptysis, chest pain and weight loss ${ }^{(8)}$, progressing to pulmonary hypertension, right ventricular failure, and possibly chronic cor pulmonale ${ }^{(9)}$. Clinical and radiological findings are frequently similar to thromboembolic disease. Due to its rarity and insidious growth pattern, PAS may be diagnosed as chronic PTE, leading to a diagnostic delay and inappropriate therapy such as anticoagulation or prolonged thrombolysis ${ }^{(10)}$

At imaging studies, PAS presents as unilateral, intravascular lobulated masses with heterogeneous contrast enhancement, that may cause vascular distension and local extravascular dissemination $^{(11)}$. Also, the lungs are frequently affected by metastases ${ }^{(7)}$. According to Yi et al. ${ }^{(12)}$, tomographic findings suggesting the diagnosis of PAS include low attenuation filling defect of the entire luminal diameter of a segment or of the whole extent of the main pulmonary artery, enlargement of the involved arteries and
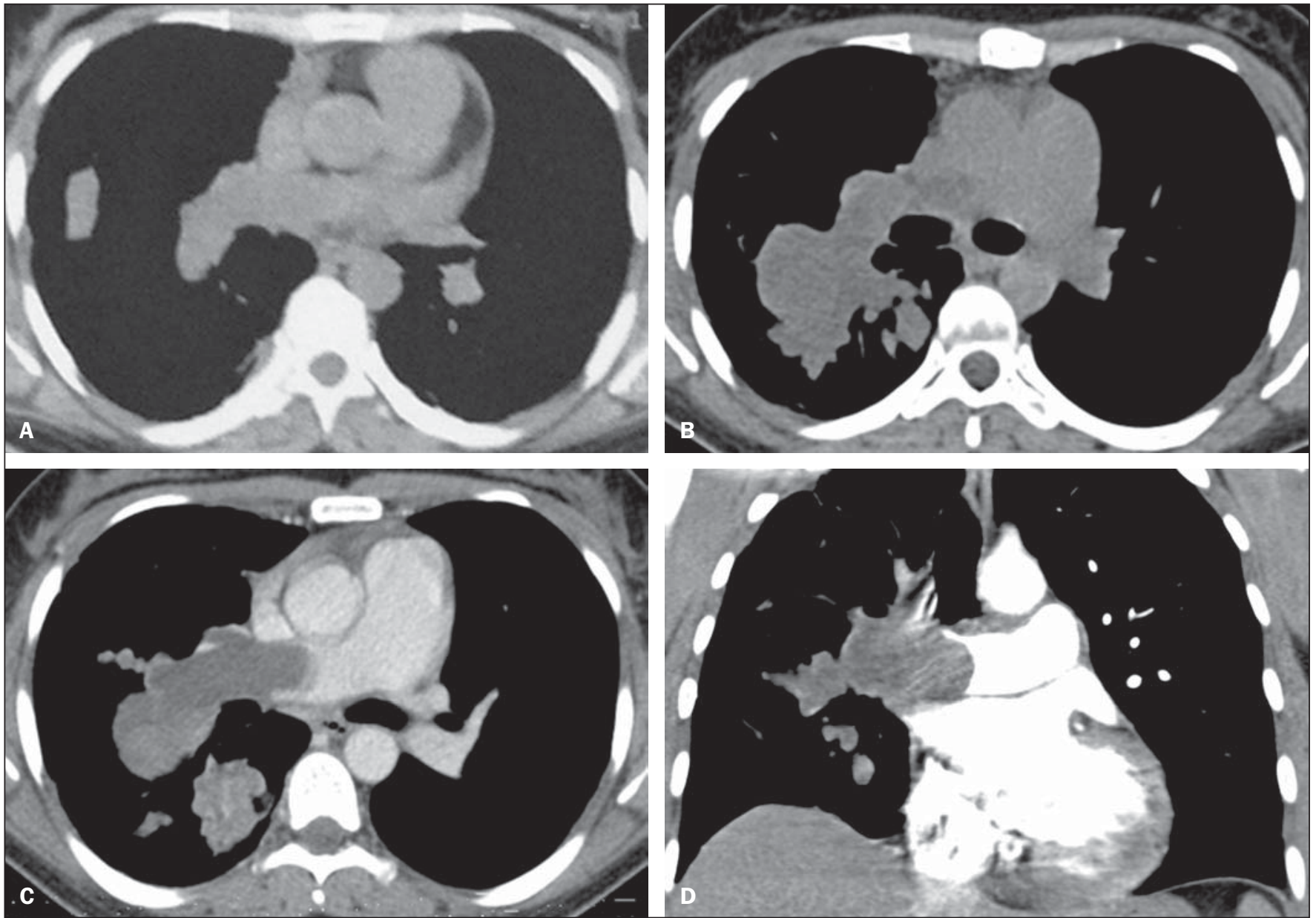

Figure 1. Axial chest computed tomography (A) demonstrating hypodense mass occupying the lumen of the right pulmonary artery. The luminal diameter is preserved. After seven months, follow-up with contrast-enhanced and non-contrast-enhanced axial (B,C) and coronal (D) computed tomography showed significant enlargement of the intraluminal mass determining dilatation of the affected vessels, with areas of contrast enhancement. 
extraluminal extension of the tumor ${ }^{(6,12)}$. The prognosis is poor, with mean survival time of approximately one year and a half after symptoms onset ${ }^{(8)}$. Due to pulmonary artery occlusion and acute symptoms, surgical resection is generally the treatment of choice ${ }^{(8)}$.

In conclusion, the present case reinforces the important role of the imaging methods in the differentiation between pulmonary artery intimal sarcoma and chronic PTE. The relevant aspects for this differentiation, such as contrast enhancement, distention of the affect vessels and extraluminal extension, allow for a correct diagnosis, avoiding delay in the required surgical approach.

\section{REFERENCES}

1. Yamanari MGI, Mansur MCD, Kay FU, et al. Bullet embolism of pulmonary artery: a case report. Radiol Bras. 2014;47:128-30.

2. Agnollitto PM, Barreto ARF, Barbieri RFP, et al. Rendu-Osler-Weber syndrome: what radiologists should know. Literature review and three cases report. Radiol Bras. 2013;46:168-72.

3. Yamada AM, Melo ALKO, Lopes GP, et al. Bilateral breast swelling secondary to superior vena cava obstruction and subclavian vein thrombosis. Radiol Bras. 2013;46:252-4.

4. Daud DF, Campos MMF, Fleury Neto LAP. Cardiac tamponade in an infant during contrast infusion through central venous catheter for chest computed tomography. Radiol Bras. 2013;46:385-6.

5. Eifer DA, Arsego FV, Torres FS. Unilateral pulmonary veins atresia: evaluation by computed tomography. Radiol Bras. 2013;46:376-8.
6. Chong S, Kim TS, Kim BT, et al. Pulmonary artery sarcoma mimicking pulmonary thromboembolism: integrated FDG PET/CT. AJR Am J Roentgenol. 2007;188:1691-3.

7. Grosse C, Grosse A. CT findings in diseases associated with pulmonary hypertension: a current review. Radiographics. 2010;30:1753-77.

8. Wong HH, Gounaris I, McCormack A, et al. Presentation and management of pulmonary artery sarcoma. Clin Sarcoma Res. 2015;5:3.

9. Dornas AP, Campos FT, Rezende CJ, et al. Intimal sarcoma of the pulmonary artery: a differential diagnosis of chronic pulmonary thromboembolism. J Bras Pneumol. 2009;35:814-8.

10. Cheng HM, Chou ASB, Chiang KH, et al. Serial CT findings of pulmonary artery intimal sarcoma in 4 months: a case report. Chin J Radiol. 2009;34:35-8.

11. Wittram C, Maher MM, Yoo AJ, et al. CT angiography of pulmonary embolism: diagnostic criteria and causes of misdiagnosis. Radiographics. 2004;24:1219-38.

12. Yi CA, Lee KS, Choe YH, et al. Computed tomography in pulmonary artery sarcoma: distinguishing features from pulmonary embolic disease. J Comput Assist Tomogr. 2004;28:34-9.

\section{Marianna Nunes Batista ${ }^{1}$, Miriam Menna Barreto ${ }^{1}$, Renata Fukamati Cavaguti ${ }^{1}$, Gláucia Zanetti ${ }^{1}$, Edson Marchiori $^{1}$}

1. Universidade Federal do Rio de Janeiro (UFRJ), Rio de Janeiro, RJ, Brazil. Mailing Address: Dr. Edson Marchiori. Rua Thomaz Cameron, 438, Valparaíso. Petrópolis, RJ, Brazil, 25685-120. E-mail: edmarchiori@gmail.com.

http://dx.doi.org/10.1590/0100-3984.2015.0046

\section{Central nervous system involvement in sarcoidosis}

Envolvimento do sistema nervoso central na sarcoidose

\section{Dear Editor,}

A 51-year-old female patient complained of mild frontotemporal headache of insidious-onset for two years. One year ago, she had an episode of focal, tonic-clonic seizures (with right lower limb paresthesia) and was prescribed carbamazepine. Cerebrospinal fluid demonstrated increased protein levels and intrathecal immunoglobulin $(\mathrm{IgG})$ synthesis, suggesting an inflammatory component. Magnetic resonance imaging was performed (Figure 1).

Sarcoidosis is a multisystem disease of unknown etiology characterized by noncaseating granulomatous inflammation ${ }^{(1)}$. There is a genetic predisposition, with T-lymphocyte receptor activation by some unknown antigen. The disease affects preferentially the respiratory system ${ }^{(1)}$. In the lungs, granulomas are observed in the interstitial compartment, showing a perilymphatic distribution along the peribronchovascular sheaths, interlobular septa and pleural surface ${ }^{(1)}$.

It is estimated that in about $5 \%$ to $15 \%$ of cases sarcoidosis affects the central nervous system. Rarely the patient presents with exclusively neurological manifestations like in the present case. Most commonly, neurosarcoidosis is observed in cases of disseminated disease $\mathrm{e}^{(2)}$.

The clinical manifestations of neurosarcoidosis are pleomorphic. Cranial nerve compromise, visual alterations, headache, weakness, paresis, paresthesia, psychiatric alterations and signs of meningeal irritation may be observed. Although rare, symp-

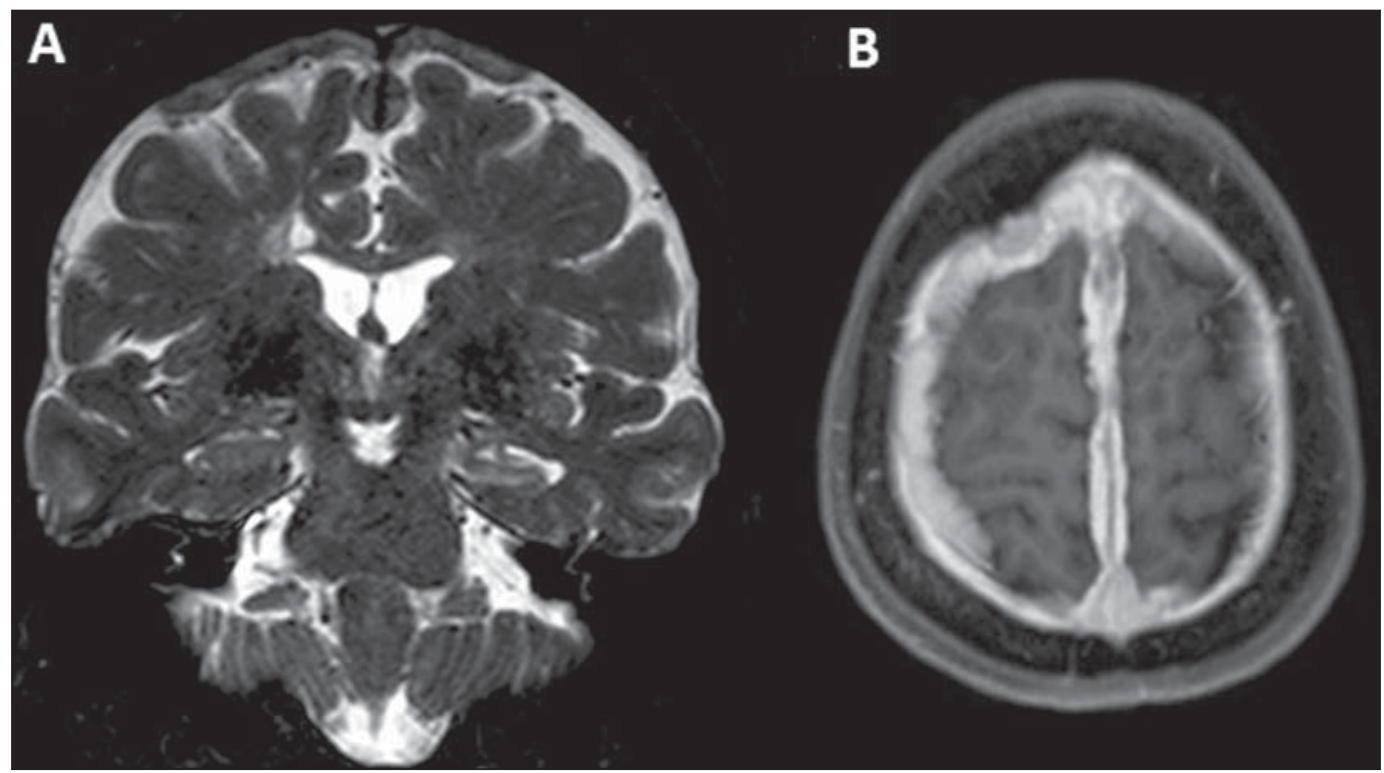

Figure 1. A: Coronal magnetic resonance imaging-T2-weighted sequence demonstrating diffuse pachymeningeal thickening most prominent at the high convexity and extending bilaterally toward the falx, with predominance of hyposignal in association with reduction in volume and hypersignal of the left hippocampus (mesial sclerosis). B: Paramagnetic contrast-enhanced axial T1-weighted sequence showing diffuse and homogeneous pachymeningeal enhancement. 